\title{
Hypertension treatment in the Asia-Pacific: the role of and treatment strategies with nebivolol
}

\author{
Cheol-Ho Kim, ${ }^{1}$ Nelson Abelardo, ${ }^{2}$ Peera Buranakitjaroen, ${ }^{3}$ Rungroj Krittayaphong, ${ }^{3}$ \\ Chin Hock Lim, ${ }^{4}$ Sung-Ha Park, ${ }^{5}$ Nguyen Vinh Pham, ${ }^{6}$ Gregorio Rogelio, ${ }^{7}$ \\ Bernard Wong, ${ }^{8}$ Lip Ping Low ${ }^{9}$
}

For numbered affiliations see end of article.

\section{Correspondence to} Professor Cheol-Ho Kim Department of Internal Medicine, Seoul National University College of Medicine, 103 Daehak-ro, Jongno-gu, Seoul 110-799, Korea; cheolkim@plaza.snu.ac.kr; cheolkim@snu.ac.kr

Received 29 June 2015 Accepted 18 January 2016

\section{CSLinked}

- http://dx.doi.org/10.1136/ heartasia-2016-010728

\section{CrossMark}

To cite: $\mathrm{Kim} \mathrm{C}-\mathrm{H}$,

Abelardo N,

Buranakitjaroen $\mathrm{P}$, et al. Heart Asia 2016;8:22-26.

doi:10.1136/heartasia-2015010656

\section{ABSTRACT}

Hypertension is a common disease, and hypertensive patients are at increased risk of cardiovascular events. The prevalence and socioeconomic burden of hypertension in the Asia-Pacific region are predicted to increase in the coming decades. Effective blood pressure lowering reduces overall cardiovascular morbidity and mortality in patients, yet doubt has been raised regarding the use of (mainly older generation) $\beta$-blockers as initial therapy in hypertension. Consequently, several international treatment guidelines do not recommend $\beta$-blockers for the treatment of hypertension. However, in contrast to first-generation and second-generation $\beta$-blockers, the third-generation, vasodilating $\beta$-blocker nebivolol has a considerably better metabolic, haemodynamic and side effect profile. In addition to providing effective blood pressure control similar to other $\beta$-blockers and drugs from other antihypertensive classes, nebivolol exerts a dual mechanism for increasing the bioavailability of the naturally occurring vasodilator nitric oxide. The clinical benefits and significance of enhancing nitric oxide levels in hypertensive patients have been shown in direct comparisons of nebivolol with other $\beta$ blockers. While $\beta$-blockers generally provide comparable blood pressure reductions, only nebivolol demonstrated enhanced vasodilation and blood flow by increasing the expression of endothelial nitric oxide synthase and therefore increasing nitric oxide release from the endothelium. In contrast to other $\beta$-blockers, therefore, it has been suggested that nebivolol has beneficial effects in several hypertensive subgroups due to its vasodilating properties. Considering the existing data, it may be timely for treatment guidelines to recommend thirdgeneration vasodilating $\beta$-blockers as a first-line option for the pharmacotherapy of hypertension.

\section{INTRODUCTION}

Hypertension is the most common health problem detected by primary healthcare physicians. ${ }^{1}$ Hypertensive patients are at an increased risk of cardiovascular events, especially heart disease, myocardial infarction and stroke, as well as kidney disease. $^{2}$ It has been shown that $47 \%$ of ischaemic heart disease and 54\% of strokes worldwide can be attributed to hypertension. ${ }^{3}$ In 2010, high blood pressure (BP) accounted for 9.4 million deaths globally, and $>1.5$ billion adults in the world are predicted to suffer from hypertension in $2025 .^{45}$ The prevalence of hypertension ranges in the Southeast Asia and Pacific regions from 5\% to $47 \%$ in men and from $7 \%$ to $38 \%$ in women, respectively, ${ }^{6}$ and is expected to further increase due to the adoption of unfavourable (Western) lifestyles and population ageing. ${ }^{7}$ Although prolonged high BP causes significant-and often irreversible-adverse health effects on various organ systems in the human body, most patients remain asymptomatic for many years. Furthermore, hypertension treatment and BP control rates in the Asia-Pacific region are generally suboptimal. ${ }^{78}$ As effective BP lowering has consistently been demonstrated to reduce overall cardiovascular morbidity and mortality, ${ }^{19}$ early detection and consequent intervention are essential. ${ }^{4}$

In 1962, based on the rationale that chest pain of ischaemic heart disease should improve by reducing catecholamine-induced effects of myocardial $\beta$-adrenoreceptor activation, Sir James Black, while at ICI pharmaceuticals (UK), made a pivotal contribution to pharmacology and clinical medicine with the development of the first $\beta$-blocker. ${ }^{10}$ Today, the class of $\beta$-blockers is typically grouped in generations. First-generation $\beta$-blockers (eg, propranolol, pindolol) are non-selective and show no vasodilation effect. Second-generation $\beta$-blockers (eg, atenolol, bisoprolol) are considered $\beta_{1}$-selective while third-generation $\beta$-blockers (eg, carvedilol, nebivolol) show additional vasodilatory properties.

Since the early 1990s, doubt has been raised regarding the use of $\beta$-blockers as initial therapy in hypertension. ${ }^{11}{ }^{12}$ However, it should be noted that most of the studies reporting unfavourable effects of $\beta$-blockers in hypertension therapy were conducted with atenolol. ${ }^{13}$ In contrast to firstgeneration and second-generation $\beta$-blockers, those of the third generation exert considerably better metabolic and haemodynamic profiles. ${ }^{14}$ Nebivolol (figure 1), as a $\beta$-blocker with $\beta_{1}$-selectivity and nitric oxide $(\mathrm{NO})$-mediated vasodilatory benefits, is of particular interest. ${ }^{2} 15$

On 22 February 2014, 10 hypertension experts from the Asia-Pacific region convened for the Advisory Panel on Nebivolol in Hypertension Care meeting in Singapore. The purpose of the meeting was to analyse the role of $\beta$-blockers in BP regulation, review the current hypertension management guidelines and develop a consensus on indications for the safe and effective use of nebivolol in hypertension care.

\section{THE $\boldsymbol{\beta}$-blocker setback in hypertension care Early evidence}

In 1992, a large study conducted by the Medical Research Council on British elderly patients found no benefit of atenolol against coronary events and only little protection against stroke. ${ }^{11}$ In 1998, a 

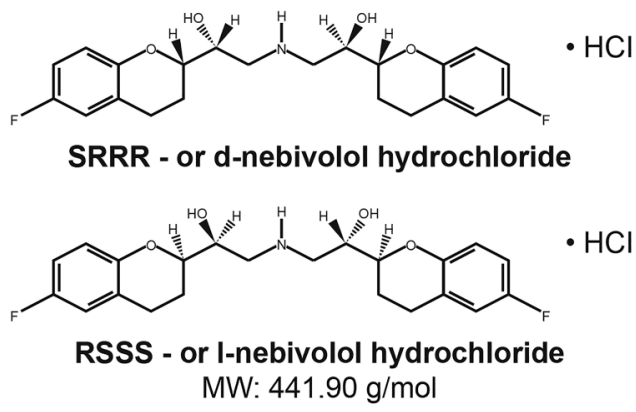

Figure 1 Structure of third-generation $\beta$-blocker nebivolol.

systematic review of trials in the elderly by Messerli et $a l^{12}$ revealed that the use of $\beta$-blockers as monotherapy in hypertension resulted in worse outcomes compared with diuretics.

\section{Recent evidence}

In 2005, a meta-analysis by Lindholm et al of 13 randomised clinical trials compared $\beta$-blockers (atenolol, metoprolol, propranolol and oxprenolol) to other hypertensive drugs in the treatment of hypertension. The authors found a $16 \%$ increase in relative risk of stroke with $\beta$-blockers compared with other drugs. In comparison to placebo or no treatment, the risk reduction for stroke using $\beta$-blockers was only about half in comparison to other antihypertensive medication. ${ }^{16}$ An increase of new-onset diabetes in $\beta$-blocker-treated patients was reported in 2007. ${ }^{17}$ Furthermore, Bangalore et al ${ }^{18}$ noticed greater reduction in heart rate was associated with higher risk of cardiovascular events. Finally, two recent large-scale hypertension trials (LIFE and ASCOT studies) demonstrated a clear superiority of newer antihypertensives over atenolol and the combination with diuretics. ${ }^{19}$ Likewise, a meta-analysis by Khan and McAlister provides no evidence for a reduction in mortality, myocardial infarction or stroke in younger patients treated with $\beta$-blockers. ${ }^{20}$ Box 1 summarises the main reasons for the lack of cardiovascular protection provided by older generation $\beta$-blockers.

\section{GUIDELINES FOR THE MANAGEMENT OF HIGH BP}

The 2014 evidence-based guidelines for the management of high BP in adults, based on a report from the panel members appointed to the Eighth Joint National Committee (JNC8), do not recommend $\beta$-blockers as first-line therapy for hypertension (Recommendation 6). The negative evaluation of $\beta$-blockers was mainly due to their unfavourable effects compared with angiotensin II receptor blocker (ARB). ${ }^{1}$ Similarly, the British Hypertension Society stated in their 2011 National Institute for

Box 1 Suggested main reasons for the lack of cardiovascular protection provided by (older) $\beta$-blockers ${ }^{21}$

- Suboptimal antihypertensive effects

- Unfavourable haemodynamics

- Reduced compliance due to many adverse effects

- Reduced effect on left ventricular hypertrophy

- Unfavourable metabolic effects (lipid and glucose metabolism)
Health and Care Excellence (NICE) guidelines that " $\beta$-blockers are not a preferred initial therapy for hypertension". However, the guidelines also indicated that $\beta$-blockers may be considered in younger people, particularly in certain subpopulations, for example, those with an intolerance or contraindication to angiotensin-converting enzyme (ACE) inhibitors and angiotensin II receptor antagonists; women of childbearing age; or people with evidence of increased sympathetic drive. ${ }^{21}$

In contrast, the Canadian guidelines recommend all drug classes as suitable for initial hypertension therapy with the exception of patients older $\geq 60$ years for whom $\beta$-blockers are not recommended. ${ }^{22}$ Likewise, in contrast to the British and American JNC8 recommendations, the 2013 European Society of Cardiology/European Society of Hypertension (ESC/ESH) guidelines recommend that ACE inhibitors, ARBs, $\beta$-blockers, calcium channel blockers (CCBs) and diuretics are all suitable for both the initiation and maintenance of antihypertensive therapy either as monotherapy or in certain combinations. ${ }^{23}$ Also in agreement with the above recommendations are hypertension guidelines from several Asian countries, for example, China, Korea, India, Indonesia and Singapore, which all consider $\beta$-blockers as a potential first-line option for hypertension. $^{8}$ Box 2 summarises the recommendations common to current hypertension guidelines.

Taken together, recommendations in certain hypertension management guidelines have been based on negative clinical data with some $\beta$-blockers, and atenolol in particular. ${ }^{13}$ The widely existing ' $\beta$-blocker phobia' is largely due to unfavourable outcome results of specific $\beta$-blockers in clinical trials, especially those of the first and second generation. However, not all $\beta$-blockers are the same. In fact, the 2009 reappraisal of the ESH/ESC guidelines highlighted that $\beta$-blockers are indeed suitable for initial therapy in hypertension and that highly $\beta_{1}$-selective blockers or those with vasodilatory effects may be preferred as first-line antihypertensive agents. ${ }^{24}$

The third-generation $\beta$-blocker nebivolol has both the highest $\beta_{1}$-cardioselectivity and vasodilatory benefits that are mediated by the L-arginine/NO pathway. As discussed in detail below, nebivolol shows similar efficacy compared with other monotherapies in hypertension treatment, often with fewer adverse

Box 2 Recommendations common to all contemporary hypertension guidelines (adapted from Jennings and Touyz (2013) with slight modifications) ${ }^{24}$

- Blood pressure (BP) is a continuous variable but for practical purposes is broken down into grades

- Measure BP carefully

- High BP is associated with adverse prognosis

- High BP is especially adverse for people who have extensive vascular disease, multiple cardiovascular risk factors, diabetes mellitus or renal disease

- Assess total cardiovascular risk

- Lifestyle measures come first when attempting to lower BP, cardiovascular risk and improving responses to antihypertensive drugs

- Drugs come next, generally initial monotherapy followed by combinations

- Combinations from different drug classes are generally good; combinations sharing similar actions are generally not advised 
effects. $^{2} 25$ In addition, the antioxidant and NO-releasing properties of nebivolol have been suggested to attenuate oxidative stress and endothelial dysfunction and thus to provide added benefits for patients receiving antihypertensive treatment. ${ }^{25}$

\section{NEBIVOLOL: MECHANISMS OF ACTION AND CLINICAL EFFICACY IN BP REGULATION}

\section{Basic pharmacokinetic and pharmacodynamic data for} nebivolol

Nebivolol is a racemic mixture made up of equal amounts of Dand L-nebivolol. Compared with other currently available $\beta$-blockers (such as metoprolol, bisoprolol and carvedilol), nebivolol is the most selective $\beta_{1}$-adrenoreceptor antagonist in the human myocardium with a 321-fold higher selectivity for $\beta_{1}$-adrenoreceptors compared with $\beta_{2}$-adrenoreceptors. ${ }^{26}$ The $\mathrm{D}$-isomer of nebivolol is mainly responsible for the selective $\beta_{1}$-adrenoreceptor antagonist activity, while the L-isomer primarily contributes to the vasodilating properties of nebivolol. ${ }^{27}$

Whereas carvedilol exerts vasodilating effects via $\alpha_{1}$-receptor blockade, nebivolol stimulates the endothelial L-arginine/NO pathway and thus increases the bioavailability of the naturally occurring vasodilator NO (figure 2). ${ }^{25}$ This effect was first demonstrated in isolated canine coronary arteries where vasodilation by nebivolol was shown to be endothelium-dependent and sensitive to blockade by NO inhibitors. ${ }^{28}$

A starting dose of nebivolol $5 \mathrm{mg}$ once daily is recommended for most patients with hypertension. ${ }^{29}$ A single dose of nebivolol allows $24 \mathrm{~h}$ control of BP, exerting a constant antihypertensive action that respects the physiological circadian rhythm. ${ }^{30} 31$ Upon oral administration, nebivolol is absorbed rapidly and unaffected by food. ${ }^{27}$ In the circulation, approximately $98 \%$ of nebivolol is protein bound. ${ }^{27}$

\section{Clinical efficacy and tolerability of nebivolol}

Several studies have clearly established that the antihypertensive efficacy of nebivolol is similar to that of other $\beta$-blockers, for example, atenolol and bisoprolol. ${ }^{32} 33$ In addition, a study has also demonstrated that intervention with nebivolol, but not with metoprolol succinate, resulted in significantly reduced central systolic and diastolic BPs, central pulse pressure and left ventricular wall thickness in hypertensive patients (aged 3065 years) treated for 1 year. ${ }^{34}$ Furthermore, whereas no significant difference has been found between bisoprolol and atenolol in terms of reduction of aortic pulse pressure after 12 weeks of treatment, ${ }^{35}$ nebivolol reduced aortic pulse pressure to a greater extent than atenolol, ${ }^{36}$ and yet affects a less pronounced impact

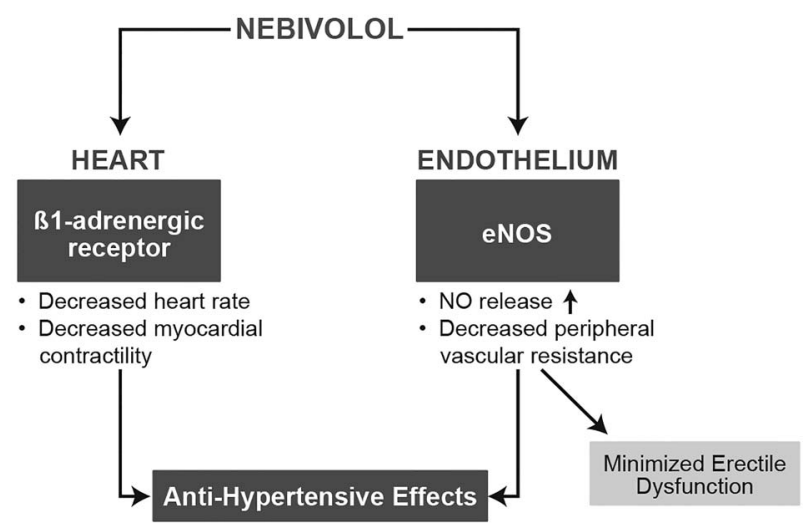

Figure 2 Dual mechanism of action for the antihypertensive effects of nebivolol. eNOS, endothelial nitric oxide synthase; NO, nitric oxide. on augmentation index (AIx). ${ }^{37}$ In fact, in contrast to most other antihypertensive drugs, the majority of B-blockers cause an increase in AIx values. ${ }^{38}$ However, recent data strongly indicate an AIx-reducing potential for nebivolol. Compared with carvedilol and metoprolol, nebivolol treatment resulted in a statistically significant reduction of heart rate-adjusted AIx values. ${ }^{39}$ Results from the Conduit Artery Function Evaluation (CAFÉ) study indicate that even a $3 \mathrm{~mm} \mathrm{Hg}$ reduction in central pulse pressure has been associated with better cardiovascular outcomes ${ }^{40}$; hence, these observations may be of substantial clinical relevance. While conventional brachial BP measurement is simple and has remained the gold standard for the measurement of BP, data on central BP are of particular interest as this parameter has been suggested to be a more accurate predictor of cardiovascular risk and response to antihypertensive therapy. ${ }^{41}$

In vivo, the clinical significance of the ability to modify NO levels has been shown in a direct comparison of nebivolol with atenolol in hypertensive patients. Whereas treatment with both $\beta$-blockers resulted in comparable BP reduction, only nebivolol enhanced vasodilation and blood flow by increasing the expression of endothelial nitric oxide synthase and therefore increasing NO release from the endothelium. ${ }^{42}$ Similarly, nebivolol, but not bisoprolol, significantly increased flow-mediated endothelial-dependent vasodilation and thus endothelial function. $^{43}$

Because of its vasodilating properties, nebivolol also offers beneficial hemodynamic effects in hypertensive patients. Unlike non-vasodilating $\beta$-blockers, nebivolol increased stroke volume at preserved cardiac output in several clinical trials. ${ }^{44} 45$ Furthermore, in contrast to bisoprolol, nebivolol does not lead to a reduction in cardiac index (ie, the ratio of cardiac output to body surface area). ${ }^{45}$ The favourable haemodynamic profile of nebivolol (ie, preservation of cardiac output and index, reduction of peripheral resistance and improved diastolic function) might thus provide hypertensive patients with clinically relevant benefits regarding the impairment of systolic and/or diastolic function. ${ }^{46}$

The efficacy and side effect profile of nebivolol has also been tested against several other antihypertensive drug classes. In trials comparing nebivolol against CCBs (eg, amlodipine), similar response rates in the BP-lowering effects between the active treatment groups were found. However, side effect rates were significantly lower for nebivolol. ${ }^{47}$ In two separate studies, the reduction of diastolic BP with nebivolol $(5 \mathrm{mg} /$ day $)$ was similar to that achieved with lisinopril $(20 \mathrm{mg} / \mathrm{day})^{48}$ but greater than that achieved with enalapril $(10 \mathrm{mg} /$ day $) .{ }^{49}$ In comparison to losartan $(50 \mathrm{mg} /$ day), the number of patients with normalised BP was significantly higher with nebivolol $(5 \mathrm{mg} /$ day $)$ after 6 weeks, although after 12 weeks, the results were similar between treatment groups. Of note, patients treated with nebivolol benefited not only from a significantly greater reduction of diastolic BP but also from a better quality of life compared with those in the losartan group. ${ }^{50}$

Support for the efficacy and favourable side effect profile of nebivolol also originates from a recent meta-analysis of 13 randomised controlled trials in which nebivolol was compared in nine studies with a variety of other antihypertensive drugs (ACE inhibitors (enalapril, lisinopril), other $\beta$-blockers (atenolol, bisoprolol, metoprolol), an ARB (losartan) and CCBs (amlodipine, nifedipine)) and in other studies with placebo. Overall, nebivolol achieved similar or better rates of treatment response and BP normalisation than drugs from other classes and other antihypertensive drugs combined, with similar tolerability to placebo and significantly better tolerability. ${ }^{51}$ Due to its vasodilating 
properties, nebivolol has shown beneficial effects in minimising erectile dysfunction in contrast to other $\beta$-blockers. ${ }^{52}$

Furthermore, disturbance of lipid metabolism, carbohydrate metabolism/insulin signalling and risk of new-onset diabetes are significantly lower in nebivolol-treated patients compared with other $\beta$-blockers. $^{2}{ }^{25}$ In the SENIORS trial, nebivolol was associated with fewer cases of new-onset diabetes mellitus than placebo (1.8\% nebivolol vs $2.1 \%$ placebo); these differences, however, were not statistically significant. ${ }^{53}$ In hypertensive patients with type 2 diabetes, treatment with nebivolol did not result in any adverse effects with respect to lipid or glycaemic control. ${ }^{54}$ In an efficacy safety study of 6356 mild-to-moderate essential hypertensive patients or patients with isolated systolic hypertension, no serious adverse events were associated with nebivolol and there were no reports of cold extremities, a common side effect of other $\beta$-blockers. ${ }^{55}$ In agreement with other reports, the study further revealed a very similar efficacy of nebivolol both as mono and add-on therapy.

\section{TREATMENT PRACTICE: WHERE DOES NEBIVOLOL FIT IN?}

As highlighted by ACCF/AHA 2011 Expert Consensus and the $\mathrm{ESH} / \mathrm{ESC}$ reappraisal in 2009, nebivolol does not exert the adverse effects commonly seen in older generation $\beta$-blockers, particularly depression, sexual dysfunction, reduced exercise capacity, as well as lipid and glucose dyshomeostasis. ${ }^{56}$ Therefore, it is important for cardiologists and general medical practitioners to be aware that $\beta$-blockers do not comprise a homogenous class, but possess distinct strengths and weaknesses that must be carefully evaluated in order to provide hypertensive subjects with patient-tailored treatment plans. In particular, physicians of hypertensive patients need to evaluate $\beta$-blockers regarding their pharmacokinetic properties (eg, lipophilic vs hydrophilic drugs, drug clearance), mechanism of action, adverse events (eg, cardiovascular, metabolic, pulmonary, central effects, sexual dysfunction), contraindications and drug interactions. Hence, each treatment strategy should be tailored based on individual circumstances, patient preferences and drug tolerability; only then are optimised BP control rates likely to be achieved. When compared with currently available $\beta$-blockers and other antihypertensive agents, nebivolol may have advantages in several patient subgroups:

- First, compelling evidence indicates that nebivolol has no negative effects on pulmonary airways and is clinically well tolerated in hypertensive patients suffering from COPD. ${ }^{57}$

- Second, due to its $24 \mathrm{~h}$ coverage, ${ }^{30} 31$ nebivolol is a good choice in cases of poor compliance.

- Third, in comparison to older $\beta$-blockers, nebivolol appears to have more favourable effects on erectile function in hypertensive men. Three months after switching to nebivolol, patients with no erectile dysfunction increased from 34\% to $50 \%$ and those with severe erectile dysfunction decreased from $18 \%$ to $5 \% .^{52}$ These favourable effects are likely associated with nebivolol's effect on the bioavailability of NO. $^{2} 58$ This makes nebivolol an ideal $\beta$-blocker for male hypertensive patients.

- Fourth, based on its dual mode of action, nebivolol may be particularly beneficial in patients with difficult-to-treat hypertension (eg, the elderly, diabetics, obese patients), in whom NO-mediated endothelial dysfunction may be more pronounced. ${ }^{25}$ In 2003, Kamp et $a l^{59}$ reported that nebivolol not only preserved cardiac output and increased stroke volume but also induced a reduction in peripheral resistance. Due to different modes of action, these vasodilating properties are not found in older generation $\beta$-blockers.

\section{CONCLUSION}

Hypertension is a major risk factor for cardiovascular diseases and other end-organ damage and is thus a leading cause of morbidity and mortality. ${ }^{1}$ The prevalence, and consequently socioeconomic burden, of hypertension in the Asia-Pacific region is predicted to increase significantly in the coming decades. ${ }^{5}$ Unfortunately, current awareness of hypertension is low and the use of antihypertensive drugs is suboptimal in many countries. Although $\beta$-blockers are generally effective in reducing elevated BP either as mono or add-on therapy, several recent reviews and guidelines, especially those published by JNC8 and the British Hypertension Society (NICE guidelines), do not recommend $\beta$-blockers as firstline antihypertensive interventions, ${ }^{1}{ }^{21}$ even though most studies on $\beta$-blockers that were included in the analysis were conducted with the second generation, only $\beta_{1}$-selective drug atenolol. ${ }^{13}$

However, as the 2013 ESC/ESH guidelines highlight, $\beta$-blockers are not all alike; in particular, vasodilating $\beta$-blockers do not share some of the limitations of traditional $\beta$-blockers. ${ }^{23}$ Among the third-generation vasodilating $\beta$-blockers, nebivolol may be particularly suitable as a first-line treatment for hypertension. The recommendation is based on clinical data, indicating that in addition to providing effective BP control similar to other $\beta$-blockers and antihypertensive drugs, nebivolol's dual mode of action for increasing the bioavailability of the vasodilator NO may be of particular benefit to hypertensive patients. ${ }^{2} 2560$

\section{Author affiliations \\ ${ }^{1}$ Department of Internal Medicine, Seoul National University, Seongnam-si, Korea ${ }^{2}$ College of Medicine, University of the Philippines College of Medicine, Metro Manila, Philippines \\ ${ }^{3}$ Department of Medicine, Siriraj Hospital, Mahidol University, Bangkok, Thailand ${ }^{4} \mathrm{C}$ H Lim Cardiology Clinic, Mount Elizabeth Medical Centre, Singapore, Singapore ${ }^{5}$ Division of Cardiology, Severance Cardiovascular Hospital—Yonsei University College of Medicine, Seoul, Korea \\ ${ }^{6}$ Tam Duc Heart Hospital, Ho Chi Minh City, Vietnam \\ ${ }^{7}$ Heart Institute, St. Luke's Medical Center Global City, Metro Manila, Philippines \\ ${ }^{8}$ Everhealth Medical Centre Limited, Hong Kong, Hong Kong \\ ${ }^{9}$ Low Cardiology Clinic, Mount Elizabeth Medical Centre, Singapore, Singapore}

Acknowledgements The authors would like to thank Ruth Schaffer of MIMS Pte Ltd for providing editorial support with collating author feedback and incorporating revisions.

Contributors All authors attended the consensus meeting and contributed to the development of consensus recommendations on use of nebivolol in hypertension. All authors reviewed and had input drafting and revising the manuscript, and approved the final version prior to submission. The manuscript is based on equal contribution of all authors present during the expert meeting: (1) pre-meeting author survey; (2) experts input during the workshop, roundtable discussions and drafting of the manuscript; (3) authors recommendation of cited references; and (4) reviewing, editing and final approval of manuscript draft.

Competing interests A. Menarini Asia-Pacific Pte Ltd provided logistic support and funding for the consensus meeting, and the authors received fair market honorarium for their time spent attending the consensus meeting and developing the manuscript. A. Menarini Asia-Pacific Pte Ltd also paid for MIMS Pte Ltd to provide editorial support. C-HK, PB, RK, CHL, S-HP and BW reports personal fees from A. Menarini Asia-Pacific Pte Ltd, during the preparation of this manuscript. NA and GR reports personal fees from A. Menarini Asia-Pacific Pte Ltd, during the preparation of this manuscript; personal fees from A. Menarini Philippines, Inc, outside the submitted work. NVP reports personal fees from A. Menarini Asia-Pacific Pte Ltd during the preparation of this manuscript, and from Servier, Sanofi, Astra Zeneca, Merck, MSD, Bayer, United Pharma International, Abbott, Pfizer, and Tedis, outside the submitted work. PLL reports personal fees from A. Menarini Asia-Pacific Pte Ltd, during the preparation of this manuscript; personal fees from Merck Serono Pharmaceuticals, outside the submitted work.

Provenance and peer review Not commissioned; externally peer reviewed.

\section{REFERENCES}

1 James PA, Oparil S, Carter BL, et al. 2014 evidence-based guideline for the management of high blood pressure in adults: report from the panel members appointed to the Eighth Joint National Committee (JNC 8). JAMA 2014;311:507-20. 
2 Wojciechowski D, Papademetriou V. Beta-blockers in the management of hypertension: focus on nebivolol. Expert Rev Cardiovasc Ther 2008;6:471-9.

3 Lawes CM, Vander Hoorn S, Rodgers A. Global burden of blood-pressure-related disease, 2001. Lancet 2008;371:1513-18.

4 Kintscher U. The burden of hypertension. Eurolntervention 2013;9(Suppl R): R12-15.

5 Kearney PM, Whelton M, Reynolds K, et al. Global burden of hypertension: analysis of worldwide data. Lancet 2005;365:217-23.

6 Chiang $\mathrm{CE}$, Chen $\mathrm{CH}$. Hypertension in the Asia-Pacific region. $J$ Hum Hypertens 2008;22:441-3.

7 Perkovic V, Huxley R, Wu Y, et al. The burden of blood pressure-related disease: a neglected priority for global health. Hypertension 2007;50:991-7.

8 Tomlinson B, Dalal JJ, Huang J, et al. The role of $\beta$-blockers in the management of hypertension: an Asian perspective. Curr Med Res Opin 2011;27:1021-33.

9 Turnbull F. Effects of different blood-pressure-lowering regimens on major cardiovascular events: results of prospectively-designed overviews of randomised trials. Lancet 2003;362:1527-35.

10 Baker JG, Hill SJ, Summers RJ. Evolution of $\beta$-blockers: from anti-anginal drugs to ligand-directed signalling. Trends Pharmacol Sci 2011;32:227-34.

11 No authors listed]. Medical Research Council trial of treatment of hypertension in older adults: principal results. MRC Working Party. BMJ 1992;304:405-12.

12 Messerli FH, Grossman E, Goldbourt U. Are beta-blockers efficacious as first-line therapy for hypertension in the elderly? A systematic review. JAMA 1998;279:1903-7.

13 Wiysonge CS, Opie LH. $\beta$-Blockers as initial therapy for hypertension. JAMA 2013:310:1851-2.

14 Chrysant SG, Chrysant GS. Current status of $\beta$-blockers for the treatment of hypertension: an update. Drugs Today (Barc) 2012;48:353-66.

15 Cheng JW. Nebivolol: a third-generation beta-blocker for hypertension. Clin Ther 2009;31:447-62.

16 Lindholm LH, Carlberg B, Samuelsson O. Should beta blockers remain first choice in the treatment of primary hypertension? A meta-analysis. Lancet 2005;366:1545-53.

17 Elliott WJ, Meyer PM. Incident diabetes in clinical trials of antihypertensive drugs: a network meta-analysis. Lancet 2007;369:201-7.

18 Bangalore S, Sawhney S, Messerli FH. Relation of beta-blocker-induced heart rate lowering and cardioprotection in hypertension. J Am Coll Cardiol 2008;52:1482-9.

19 De Caterina AR, Leone AM. Why beta-blockers should not be used as first choice in uncomplicated hypertension. Am J Cardiol 2010;105:1433-8.

20 Khan N, McAlister FA. Re-examining the efficacy of beta-blockers for the treatment of hypertension: a meta-analysis. CMAJ 2006;174:1737-42.

21 National Clinical Guideline Centre (UK). Hypertension: The Clinical Management of Primary Hypertension in Adults: Update of Clinical Guidelines 18 and 34 [Internet]. 2011. http://www.ncbi.nlm.nih.gov/books/NBK83274/ accessed 21 Apr 2014).

22 Dasgupta K, Quinn RR, Zarnke KB, et al. The 2014 Canadian Hypertension Education Program recommendations for blood pressure measurement, diagnosis, assessment of risk, prevention and treatment of hypertension. Can J Cardiol 2014:30:485-501.

23 Mancia G, Fagard R, Narkiewicz K, et al. 2013 ESH/ESC practice guidelines for the management of arterial hypertension. Blood Press 2014;23:3-16.

24 Mancia G, Laurent S, Agabiti-Rosei E, et al. Reappraisal of European guidelines on hypertension management: a European Society of Hypertension Task Force document. J Hypertens 2009;27:2121-58.

25 Toblli JE, DiGennaro F, Giani JF, et al. Nebivolol: impact on cardiac and endothelial function and clinical utility. Vasc Health Risk Manag 2012:8:151-60.

26 Bristow MR, Nelson P, Minobe W, et al. P-121: Characterization of $\beta 1$-adrenergic receptor selectivity of nebivolol and various other beta-blockers in human myocardium. Am J Hypertens 2005;18(Suppl. 4):51A-2A.

27 Moen MD, Wagstaff AJ. Nebivolol: a review of its use in the management of hypertension and chronic heart failure. Drugs 2006;66:1389-409; discussion 410.

28 Gao YS, Nagao T, Bond RA, et al. Nebivolol induces endothelium-dependent relaxations of canine coronary arteries. J Cardiovasc Pharmacol 1991;17:964-9.

29 Nebilet [Concise Prescribing Information]. Luxembourg: Menarini International O.L. S.A, 2006.

30 Lacourciere Y, Poirier L, Lefebvre J, et al. Comparative assessment of antihypertensive efficacy of DL-nebivolol and D-nebivolol in patients with confirmed mild to moderate hypertension. J Cardiovasc Pharmacol 1995;25:619-24.

31 Van Nueten L, Dupont AG, Vertommen C, et al. A dose-response trial of nebivolol in essential hypertension. J Hum Hypertens 1997;11:139-44.

32 Van Nueten L, Taylor FR, Robertson Jl. Nebivolol vs atenolol and placebo in essential hypertension: a double-blind randomised trial. J Hum Hypertens 1998:12:135-40.

33 Czuriga I, Riecansky I, Bodnar J, et al. Comparison of the new cardioselective beta-blocker nebivolol with bisoprolol in hypertension: the Nebivolol, Bisoprolol Multicenter Study (NEBIS). Cardiovasc Drugs Ther 2003;17:257-63.
34 Kampus P, Serg M, Kals J, et al. Differential effects of nebivolol and metoprolol on central aortic pressure and left ventricular wall thickness. Hypertension 2011;57:1122-8.

35 Park S, Rhee MY, Lee SY, et al. A prospective, randomized, open-label, active-controlled, clinical trial to assess central haemodynamic effects of bisoprolol and atenolol in hypertensive patients. J Hypertens 2013;31:813-19.

36 Dhakam Z, Yasmin, McEniery CM, et al. A comparison of atenolol and nebivolol in isolated systolic hypertension. J Hypertens 2008;26:351-6.

37 Redón J, Pascual-Izuel JM, Rodilla E, et al. Effects of nebivolol and atenolol on central aortic pressure in hypertensive patients: a multicenter, randomized, double-blind study. Blood Press 2014;23:181-8.

38 Doumas $M$, Gkaliagkousi $E$, Katsiki N, et al. The effect of antihypertensive drugs on arterial stiffness and central hemodynamics: Not all fingers are made the same. Open Hypertens J 2013;5(Suppl 1:M5):75-81.

39 Studinger $\mathrm{P}$, Tabak AG, Chen $\mathrm{CH}$, et al. The effect of low-dose carvedilol, nebivolol, and metoprolol on central arterial pressure and its determinants: a randomized clinical trial. J Clin Hypertens (Greenwich) 2013;15:910-17

40 Williams B, Lacy PS, Thom SM, et al. Differential impact of blood pressure-lowering drugs on central aortic pressure and clinical outcomes: principal results of the Conduit Artery Function Evaluation (CAFE) study. Circulation 2006;113:1213-25.

41 Williams B, Lacy PS. Central aortic pressure and clinical outcomes. J Hypertens 2009;27:1123-5.

42 Tzemos N, Lim PO, MacDonald TM. Nebivolol reverses endothelial dysfunction in essential hypertension: a randomized, double-blind, crossover study. Circulation 2001:104:511-14.

43 Simova II, Todorova-Konstantinova RR, Denchev SV. Effects of nebivolol versus bisoprolol on endothelial function in hypertensive patients. Exp Clin Cardio 2009;14:45-9.

44 Triposkiadis F, Giamouzis $G$, Kelepeshis $G$, et al. Acute hemodynamic effects of moderate doses of nebivolol versus metoprolol in patients with systolic heart failure. Int J Clin Pharmacol Ther 2007:45:71-7.

45 Brett SE, Forte P, Chowienczyk PJ, et al. Comparison of the effects of nebivolol and bisoprolol on systemic vascular resistance in patients with essential hypertension. Clin. Drug Investig. 2002;22:355-9.

46 Patrascu N. Diastolic heart failure in hypertension: possible preventive benefits of nebivolol beyond lowering blood pressure. Maedica (Buchar) 2013;8:285-9.

47 Mazza A, Gil-Extremera B, Maldonato A, et al. Nebivolol vs amlodipine as first-line treatment of essential arterial hypertension in the elderly. Blood Press 2002;11:182-8

48 Rosei EA, Rizzoni D, Comini S, et al. Evaluation of the efficacy and tolerability of nebivolol versus lisinopril in the treatment of essential arterial hypertension: a randomized, multicentre, double-blind study. Blood Press Supp/ 2003;1:30-5.

49 Van Nueten L, Schelling A, Vertommen C, et al. Nebivolol vs enalapril in the treatment of essential hypertension: a double-blind randomised trial. J Hum Hypertens 1997;11:813-19.

50 Van Bortel LM, Bulpitt CJ, Fici F. Quality of life and antihypertensive effect with nebivolol and losartan. Am J Hypertens 2005;18:1060-6.

51 Van Bortel LM, Fici F, Mascagni F. Efficacy and tolerability of nebivolol compared with other antihypertensive drugs: a meta-analysis. Am J Cardiovasc Drugs 2008:8:35-44.

52 Doumas M, Tsakiris A, Douma S, et al. Beneficial effects of switching from beta-blockers to nebivolol on the erectile function of hypertensive patients. Asian J Androl 2006;8:177-82.

53 Del Sindaco D, Tinti MD, Monzo L, et al. Clinical and economic aspects of the use of nebivolol in the treatment of elderly patients with heart failure. Clin Interv Aging 2010;5:381-93

54 Peter $\mathrm{P}$, Martin U, Sharma A, et al. Effect of treatment with nebivolol on parameters of oxidative stress in type 2 diabetics with mild to moderate hypertension. J Clin Pharm Ther 2006;31:153-9.

55 Cleophas TJ, Agrawal R, Lichtenthal A, et al. Nationwide efficacy-safety study of nebivolol in mildly hypertensive patients. Am J Ther 2006;13:192-7.

56 Aronow WS, Fleg JL, Pepine CJ, et al. ACCF/AHA 2011 expert consensus document on hypertension in the elderly: a report of the American College of Cardiology Foundation Task Force on Clinical Expert Consensus Documents. Circulation 2011;123:2434-506.

57 Dal Negro R. Pulmonary effects of nebivolol. Ther Adv Cardiovasc Dis 2009;3:329-34.

58 Brixius $K$, Middeke $M$, Lichtenthal $A$, et al. Nitric oxide, erectile dysfunction and beta-blocker treatment (MR NOED study): benefit of nebivolol versus metoprolol in hypertensive men. Clin Exp Pharmacol Physiol 2007;34:327-31.

59 Kamp 0, Sieswerda GT, Visser CA. Comparison of effects on systolic and diastolic left ventricular function of nebivolol versus atenolol in patients with uncomplicated essential hypertension. Am J Cardiol 2003;92:344-8.

60 Bakris GL, Basile JN, Giles TD, et al. The role of nitric oxide in improving endothelial function and cardiovascular health: focus on nebivolol. Am J Med 2010;123(Suppl 1):S2-8. 